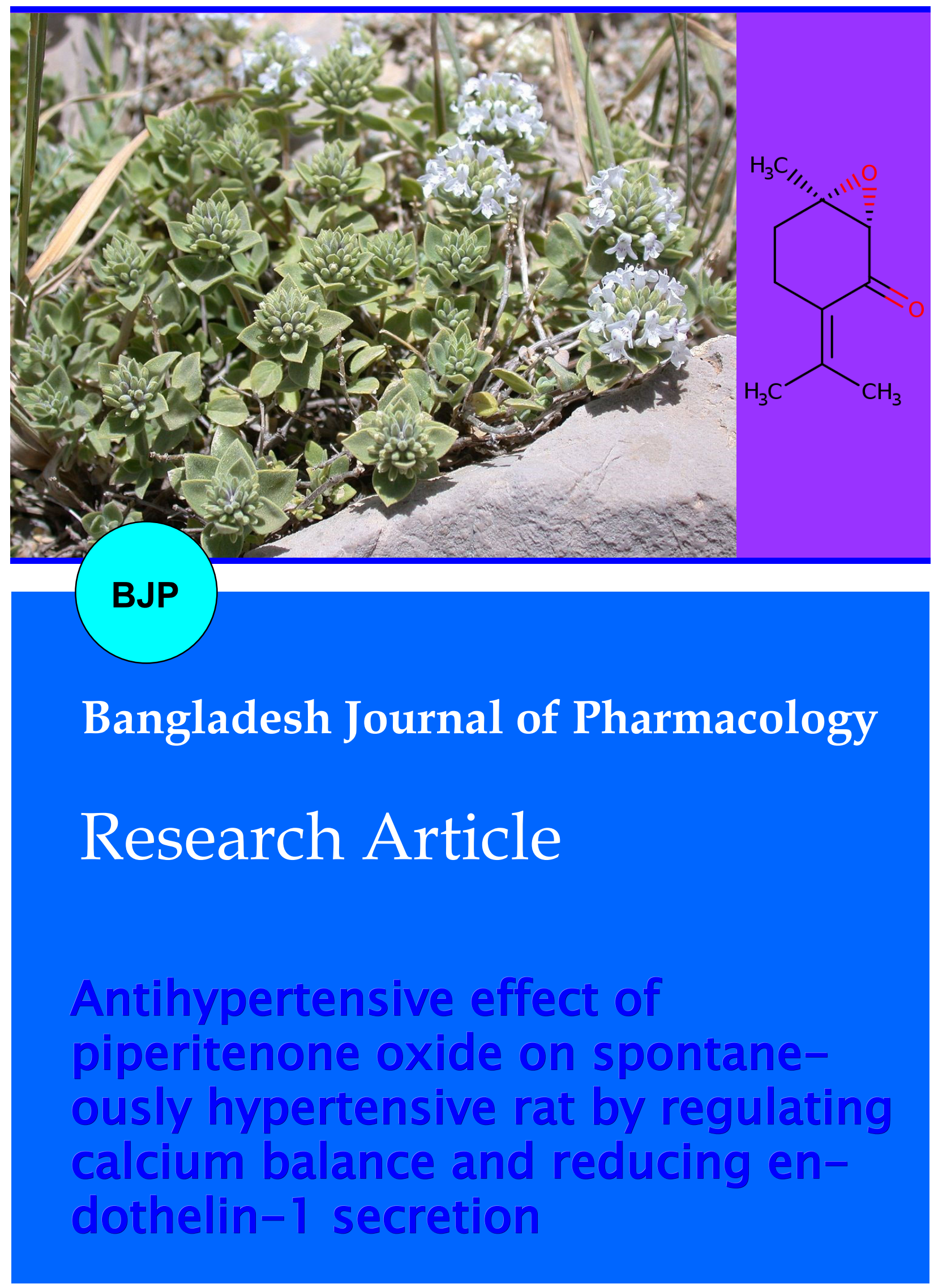


Abstracted/indexed in Academic Search Complete, Asia Journals Online, Bangladesh Journals Online, Biological Abstracts, BIOSIS Previews, CAB Abstracts, Current Abstracts, Directory of Open Access Journals, EMBASE/Excerpta Medica, Global Health, Google Scholar, HINARI (WHO), International Pharmaceutical Abstracts, Open J-gate, Science Citation Index Expanded, SCOPUS and Social Sciences Citation Index;

ISSN: $1991-0088$

\title{
Antihypertensive effect of piperitenone oxide on spontaneously hypertensive rat by regulating calcium balance and reducing endothelin-1 secretion
}

\author{
Wei Lan', Hong Ping Zhang², Ying Wang², Min Jiang², Qian Li² and Dongqing An \\ ${ }^{1}$ The Department of Chinese Materia Medica, ${ }^{2}$ The Affiliated Hospital of Traditional Chinese Medicine, Xinjiang \\ Medical University, Urumqi 830 011, XinJiang, China.
}

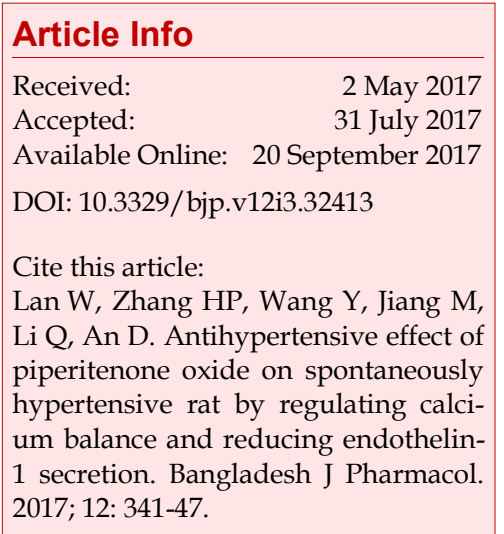

\section{Article Info}

Received:

Available Online: 20 September 2017

DOI: 10.3329/bjp.v12i3.32413

Cite this article:

Li Q, An D. Antihypertensive effect of piperitenone oxide on spontaneously um balance and reducing endothelin2017; 12: 341-47.

\begin{abstract}
The purpose of this study was to investigate the antihypertensive mechanisms of piperitenone oxide in spontaneously hypertensive rat (SHR). Compared to control SHR, piperitenone oxide reduced the systolic blood pressure and serum endothelium-1 (ET-1). The nitric oxide (NO) production and ET-1 secretion were significantly inhibited in the noradrenaline-exposed bovine aortic endothelial cells. Piperitenone oxide showed endothelium-independent but concentration-dependent relaxation of the artery rings. The responsible mechanisms appear to be related to the modulation of ET-1 and NO levels. The vasorelaxant effect of piperitenone oxide is independent of endotheliumderived mediators, which is associated with interfering intracellular calcium homeostasis by blocking $\mathrm{Ca}^{2+}$ influx, as well as intracellular $\mathrm{Ca}^{2+}$ release.
\end{abstract}

\section{Introduction}

Hypertension is an important risk factor for the cardiovascular disease, cerebrovascular disease and kidney failure worldwide, causing an estimated 7.5 million deaths per year (World Health Organization, 2009). The prevalence of hypertension is $33.5 \%$ or an estimated total of 330 million patients among the Chinese adults in 2010 (Li et al., 2012). Patients with resistant hypertension who are receiving appropriate medical therapy have high rates of cardiovascular complications, with a few treatment options (Bhatt et al., 2014).

The treatment of hypertension can be completed in the way of pharmacological and non-pharmacological therapies, such as moderation of dietary sodium intake, body-weight reduction, and increase in the physical activity. In recent years, interest in using folk medicinal plants to prevent and heal various cardiovascular problems including hypertension has grown. Thus, identifying the bioactive components of the traditional antihypertensive plants may provide health benefits for hypertensive patients.

The Uyghur people of Xinjiang, a region in the West of China, have developed the Traditional Uyghur Medicine, which slightly differs from the Traditional Chinese Medicine. Ziziphora clinopodioides Lam. is traditionally used to treat hypertension and heart disease in Uighur traditional medicine (Editorial board of Flora Xinjiangensis, 2004). Piperitenone oxide, identified as a monoterpenoid ketone, is a major chemical constituent of the essential oil from Z. clinopodioides and Mentha $x$ villosa (Verdian, 2008). Our previous study has shown that the intragastric treatment of spontaneously hypertensive rat (SHR) with volatile oil of $Z$. clinopodioides induces an immediate and dose-dependent decrease in the mean aortic pressure (Guo et al., 2014).

Piperitenone oxide reportedly has intestinal myorelaxant and antispasmodic effects in animal organ model (Sousa et al., 1997), in which analgesia is induced using 
either acetic acid-induced writhing or hot-plate tests, an effect that unrelated to opioid mechanism as it is unaffected by naloxone (Sousa et al., 2009). In addition, previous pharmacological investigations have determined hypotensive activities due to piperitenone oxide in the pentobarbital-anesthetized rat (Lahlou et al., 2001). However, no investigation has been undertaken to examine the mechanism.

This study aimed to examine the antihypertensive effect of piperitenone oxide on SHR and to explore novel vasodilators from the Traditional Uyghur Medicine. SHR and the organ-bath technique were used to examine the hypotensive and vasodilating effects of piperitenone oxide on isolated rat thoracic aorta and investigated the underlying mechanisms.

\section{Materials and Methods}

\section{Reagents and solutions}

DAF-2 DA fluorescent dye was purchased from the Calbiochem (Germany). A sensitive ELISA kit was obtained from the Assay Designs Inc. (Ann Arbor, USA). Piperitenone oxide standard [p-menth-4(8)-en-3one,1,2-epoxy-(6Cl, 7Cl); $\mathrm{C}_{10} \mathrm{H}_{14} \mathrm{O}_{2} ; 95 \%$, w/w, HPLC grade] was obtained from the National Institute for the Control of Pharmaceutical and Biological Products (China). Phenylephrine, acetylcholine, $1 \mathrm{H}-[1,2,4]-$ oxadiazole-[4,3-a]-quinoxalin-1-one, tetraethylammonium, glibenclamide, dimethyl sulfoxide (DMSO), forskolin and ethylene glycol tetraacetic acid (EGTA) were obtained from the Sigma. Except for forskolin and glibenclamide, which were dissolved in a mixture of DMSO, all tested samples were dissolved in distilled water. Other biochemical reagents were from Sagon (China). All chemical reagents were at least analytical grade.

\section{Animals and cells}

Male 12-week-old SHR and age-matched normotensive Wistar-Kyoto rat and Sprague-Dawley rat were obtained from the Xinjiang Medical University (China). Rats were housed in a room maintained on a 12 hours light/ dark cycle under constant temperature and allowed free access to food and water. The bovine aortic endothelial cells (BAEC) in primary culture was purchased from the Cell Applications Company (USA).

\section{Vascular reactivity}

As previously described (Zhang et al., 2013a), thoracic aorta from the euthanized Sprague-Dawley rat $(60 \mathrm{mg}$ / $\mathrm{kg}$ i.p. pentobarbital sodium) was removed, cleaned of adherent tissues, and cut into 2-3 mm rings. Each ring was fixed horizontally under a resting tension of $2 \mathrm{~g}$ in a $10 \mathrm{~mL}$ organ bath filled with Krebs-Henseleit solution (pH 7.4; $130 \mathrm{mM} \mathrm{NaCl}, 4.7 \mathrm{mM} \mathrm{KCl}, 1.18 \mathrm{mM} \mathrm{KH}_{2} \mathrm{PO}_{4}$,

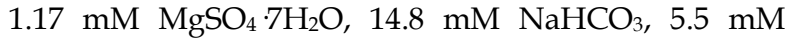
dextrose, $0.03 \mathrm{mM} \mathrm{CaNa}{ }_{2} \mathrm{EDTA}$, and $1.6 \mathrm{mM} \mathrm{CaCl}_{2}$ ). The solution was continuously kept at $37^{\circ} \mathrm{C}$ and gassed with $95 \% \quad \mathrm{O}_{2}$ and $5 \% \quad \mathrm{CO}_{2}$ for isometric tension recording. After $90 \mathrm{~min}$ of equilibration, the presence of functional endothelium in thoracic aorta from the Sprague-Dawley rat was verified according to the method described previously (Ling et al., 2010).

\section{Experimental protocols}

\section{Effect on systolic blood pressure}

The systolic blood pressures of male 12-week-old SHR and age-matched normotensive Wistar-Kyoto rat were measured using a tail cuff (Letica 5100, PanLab, Spain) after oral administration of piperitenone oxide.

\section{Assay of ET-1 secretion by norephinephrine-exposed BAEC}

BAECs in primary culture (Cell Applications, USA) were grown in EGM-2 (endothelial cell growth medium) as described previously (Montagnani et al., 2001) and were used between the passages three and five. For ELISA assays, BAECs were serum starved overnight with EBM-A (red phenol-free endothelial basal medium from the Clonetics supplemented with $1 \%$ platelet-deprived horse serum) before initiation of the experiment. BAECs were induced by 0.1 or 0.2 $\mu \mathrm{mol} / \mathrm{L}$ norephinephrine excluding control group for 24 hours before the supernatant collection. Medium ET1 levels were analyzed with a sensitive ELISA kit (Assay Designs, USA) according to the manufacturer's instruction.

\section{Assay of NO production by norephinephrine-exposed BAECS}

To analyze the NO production (Havenga et al., 2001), the prepared BAECs were incubated with $0.2 \mu \mathrm{mol} / \mathrm{L}$ norephinephrine in control group and $0.2 \mu \mathrm{mol} / \mathrm{L}$ norephinephrine plus 50 or $100 \mu \mathrm{mol} / \mathrm{L}$ piperitenone oxide in piperitenone oxide treatment group for 24 hours before $5 \times 10^{5}$ cells were harvested. The cells were transferred to the aseptic flow cytometric tubes, and washed twice with phosphate-buffered saline (PBS). The prepared cells were resuspended in $10 \mathrm{pM}$ DAF-2 DA (Calbiochem, Germany) solution and incubated at $37^{\circ} \mathrm{C}$ for 5 hours. Subsequently, the cells were harvested, washed twice with PBS, and analyzed with a flow cytometer (FACSCalibur, USA). The percentage of NO positive cells was calculated through FL1 channel.

\section{Endothelium-dependent detection}

To assess the effect of endothelium on piperitenone oxide-induced relaxation in phenylephrine $(1 \mu \mathrm{M})$, procontracted aorta rings with endothelium (+endo) and without endothelium (-endo), piperitenone oxide was added cumulatively $(100,300,500,700$, and $900 \mathrm{mg} / \mathrm{L})$ 
to construct a concentration-response curve. Finally, forskolin $(1 \mu \mathrm{M})$ was added to reach the maximum relaxation, and as diastolic as $100 \%$, recording the concentration relaxation curve (Zhang et al., 2013b).

\section{Role of three type of $\mathrm{K}^{+}$channels in piperitenone oxide-} induced relaxation

To measure the involvement of $\mathrm{K}^{+}$channels in piperitenone oxide-induced relaxation, the large conductance calcium-activated $\mathrm{K}^{+}$channel blocker $\mathrm{BaCl}_{2}$ $(100 \mu \mathrm{M})$, the voltage-dependent $\mathrm{K}^{+}$channel blocker tetraethyl ammonium (TEA, $\mathrm{mM}$ ), and the nonspecific ATP-sensitive $\mathrm{K}^{+}$channel blocker glibenclamide (100 $\mu \mathrm{M})$ were applied to endothelium-denuded rings for 30 min prior to pre-contraction by phenylephrine $(1 \mu \mathrm{M})$. The cumulative concentration-response curves of piperitenone oxide were then constructed and compared with those obtained in rings untreated with these inhibitors (Xue et al., 2011).

\section{Effect on extracellular calcium-induced contraction}

After equilibration (30-40 $\mathrm{min}$ ) and preperfusion in a $\mathrm{Ca}^{2+}$-free Krebs solution (containing $100 \mu \mathrm{M}$ EGTA and $60 \mathrm{mM} \mathrm{K}^{+}$) for $15 \mathrm{~min}, \mathrm{CaCl}_{2}$ was added cumulatively $(0.4,0.8,1.2,1.6,2.0$, and $2.4 \mathrm{mM})$ to construct a concentration-response curve. The contractile response to $\mathrm{CaCl}_{2}$ was expressed as a percentage of the maximal contraction induced by $\mathrm{K}^{+}(60 \mathrm{mmol} / \mathrm{L})$ in standard Krebs solution. In the control group, the $\mathrm{EC}_{50}$ of the extract was applied for $10 \mathrm{~min}$ before $\mathrm{KCl}$ addition (Senejoux et al., 2012).

\section{Effect on intracellular calcium release}

To determine whether piperitenone oxide inhibits the $\mathrm{Ca}^{2+}$ release from intracellular stores, endothelium-free rings were exposed to modified Krebs-Henseleit solution, and then $\mathrm{Ca}^{2+}$-free Krebs solution was changed. Soon afterward, stimulation with phenylephrine $(1 \mu \mathrm{M})$ was performed, and a vasoconstriction curve was obtained. Thereafter, for the refilling of intracellular $\mathrm{Ca}^{2+}$ stores, endothelium-free aortic rings were washed twice for $45 \mathrm{~min}$ with a standard Krebs-Henseleit solution, followed by twice washing with calcium-free Krebs-Heinseleit solution (containing $100 \mu \mathrm{M}$ EGTA). The rings were then pre-incubated with piperitenone oxide at the $\mathrm{EC}_{50}$ for $10 \mathrm{~min}$ before phenylephrine $(1$ $\mu \mathrm{M})$ was added to evoke the second transient contraction. The ratio of the second contraction to the first was calculated (Zhang et al., 2010).

\section{Statistical analysis}

Quantitative data were expressed as the mean \pm SD. Data were analyzed using OriginPro 8.0 statistical software. Student's two-tailed t-test was used for all pairwise comparisons. Values of $p<0.05$ or $p<0.01$ were considered statistically significant in all cases.

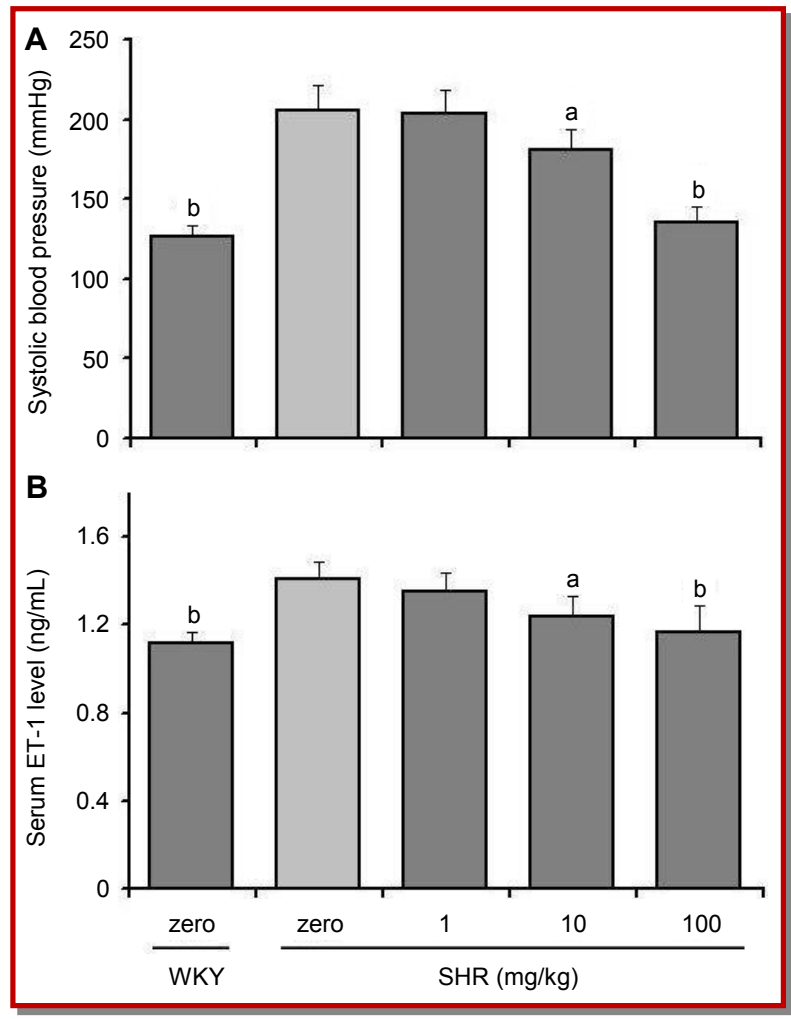

Figure 1: Effect of piperitenone oxide on (A) systolic blood pressure and (B) serum endothelin-1 (ET-1) level in spontaneously hypertensive rat (SHR). The systolic blood pressure of male 12-wk-old SHR or age-matched normotensive WistarKyoto (WKY) rat was measured using a tail cuff. SHR animals were treated twice two weeks with or without (control) oral administration of piperitenone oxide. Data are expressed as mean $\pm S D(n=8) ;{ }^{a}<<0.05$ and ${ }^{b} p<0.01$ compared to control

\section{Results}

\section{SHR and WKY animal experiments}

The systolic blood pressures of SHR administered with oral piperitenone oxide at $0,1,10$, and $100 \mathrm{mg} / \mathrm{kg}$ were $205.8 \pm 12.4$ (control), $203.1 \pm 7.9$ ( $>>0.05$, vs. control), $180.7 \pm 8.5$ ( $p<0.05$, vs. control), and $145.8 \pm 7.7(p<0.01$, vs. control) $\mathrm{mmHg}$, respectively. Without oral piperitenone oxide administration, the systolic blood pressure of normotensive Wistar-Kyoto rat was $126.2 \pm 6.3$ $\mathrm{mmHg}(\mathrm{p}<0.01$, vs. control) (Figure 1).

The SHR and Wistar-Kyoto rats were also used to confirm whether piperitenone oxide impaired the serum ET-1 level in vivo. Results demonstrated that the serum ET-1 level in SHR without oral administration of piperitenone oxide was $1.4 \pm 0.1 \mathrm{ng} / \mathrm{mL}$ (control), which was higher than that in Wistar-Kyoto rats without oral administration of piperitenone oxide $(p<0.01)$. Moreover, oral piperitenone oxide can significantly impair the serum ET-1 level in the SHR in 

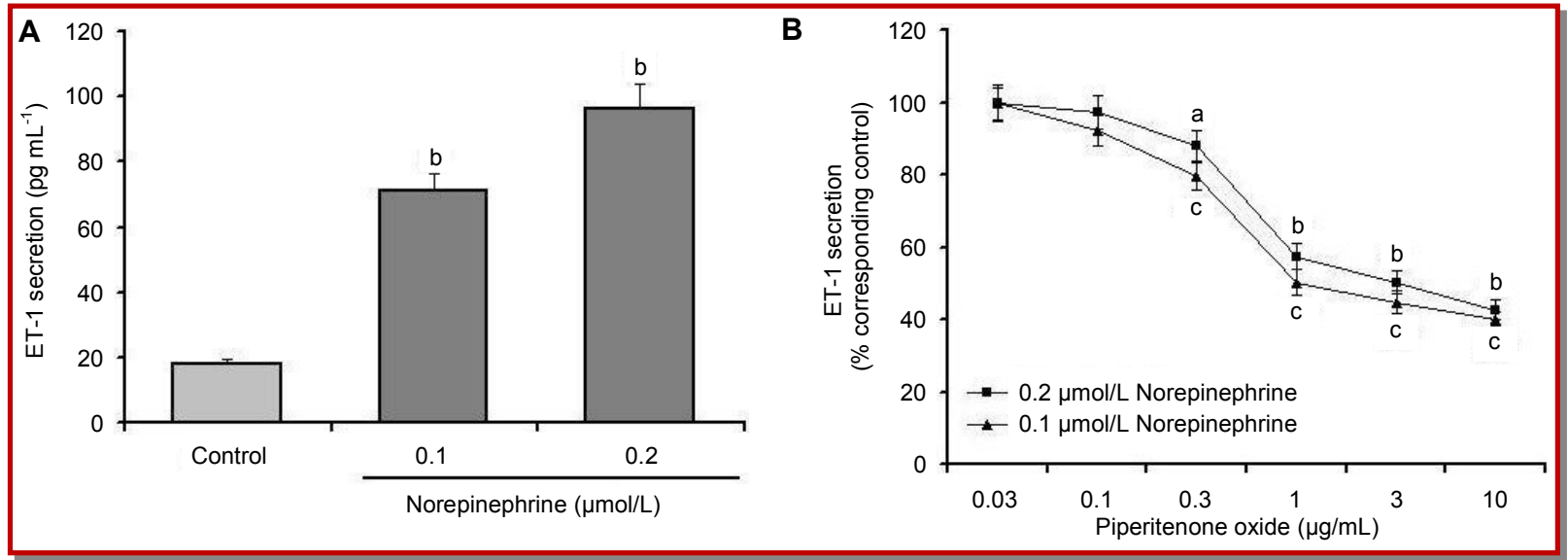

Figure 2: Effect of piperitenone oxide on endothelin-1 (ET-1) secretion by norepinephrine (NE)-exposed bovine aortic endothelial cell (BAEC). (A) BAEC was incubated with or without (control) NE for 24 hours. Compared to control, ET-1 secretion by BAEC was significantly augmented by 0.1 or $0.2 \mu \mathrm{M} \mathrm{NE}$. (B) BAEC was incubated with NE $(0.1$ or $0.2 \mu \mathrm{M}$, corresponding control) or NE $(0.1$ or $0.2 \mu \mathrm{M})$ plus piperitenone oxide ranging from 0.03 to $10 \mu \mathrm{g} / \mathrm{mL}$ for 24 hours. Data are expressed as mean \pm SD ( $\mathrm{n}=8$ ); a $\mathrm{p}<0.05$ and $\mathrm{b}, \mathrm{p} p<0.01$ compared to control or corresponding control

a dose-dependent manner (Figure 1).

\section{Assay of ET-1 secretion}

This study examined the piperitenone oxide effect on ET-1 secretion by norepinephrine-exposed BAECs. Data showed that the normal medium ET-1 level was $18.2 \pm$ $1.1 \mathrm{pg} / \mathrm{mL}$ (control). Furthermore, $0.1 \mu \mathrm{M}$ ( $\mathrm{p}<0.01$, vs. control) or $0.2 \mu \mathrm{M}$ norepinephrine $(\mathrm{p}<0.01$, compared with control) significantly augmented ET-1 secretion by BAECs (Figure 2), which was attenuated by piperitenone oxide in a dose-dependent manner, at $\mathrm{IC}_{50}=2.5$ and $4.0 \mu \mathrm{g} / \mathrm{mL}$, respectively (Figure 2).

\section{Assay of NO production}

To evaluate whether piperitenone oxide elevated NO

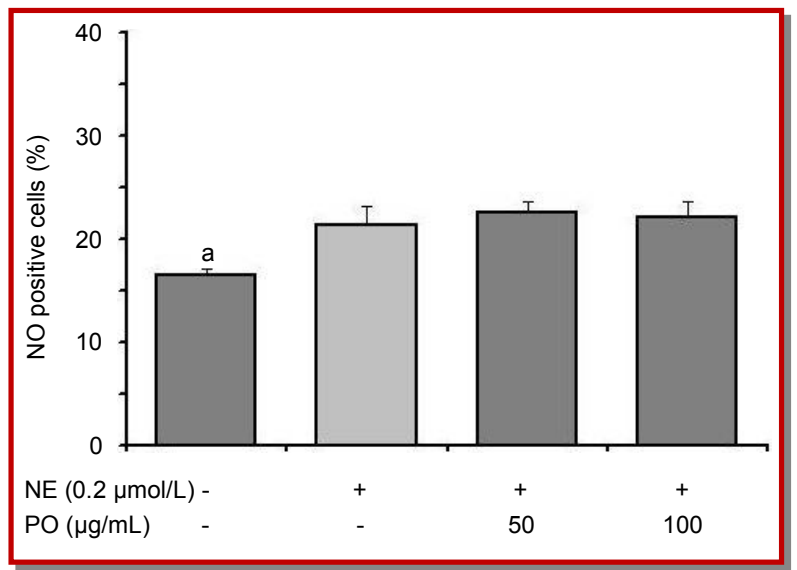

Figure 3: Effect of piperitenone oxide (PO) on nitric oxide (NO) production by norepinephrine (NE)-exposed bovine aortic endothelial cells (BAEC). BAEC was incubated with NE (control) or NE plus PO for 24 hours. The NO value detected by using flow cytometry in triplicate was expressed as NO positive cells percentage. Data are expressed as mean \pm SD $(n=3)$; ${ }^{a}<<0.05$ compared to control production by norepinephrine-exposed endothelial cells, flow cytometry was used to measure the intracellular NO level as an indicator of medium NO level. Data showed that although NO production by BAEC can be stimulated by norepinephrine, piperitenone oxide at $\leq 100 \mu \mathrm{g} / \mathrm{mL}$ did not further influence NO production by norepinephrine-exposed BAECs (Figure 3). This finding suggested that NO may not participate in the mechanism of piperitenone oxide against norepinephrine-exposed vasoconstriction.

\section{Endothelium-dependent detection}

Figure 4 shows the effect of endothelium on piperitenone oxide-induced relaxation in phenylepinephrine $(1$ $\mu \mathrm{M})$ pro-contracted aorta rings with endothelium (+endo) or without endothelium (-endo). The maximum percentage of relaxation $\left(E_{\max }\right)$ induced by piperitenone oxide in intact or denuded aortic rings was 96.7 $\pm 3.9 \%$ and $98.1 \pm 3.7 \%$, and the piperitenone oxideproduced $50 \%$ of $\mathrm{E}_{\max }\left(\mathrm{EC}_{50}\right)$ in denuded aortic rings was $219.7 \mathrm{mg} / \mathrm{L}$. All in a concentration-dependent relaxation of with endothelium was not significant difference with the group of without endothelium.

\section{Role of $\mathrm{K}^{+}$channels in relaxation}

To assess whether potassium channels interfere with the relaxation induced by piperitenone oxide, aortic rings were incubated with glibenclamide, TEA, and $\mathrm{BaCl}_{2}$ prior to contraction with phenylephrine. As shown in Figure 4, they did not affect the relaxant effect of piperitenone oxide. Each $\mathrm{K}^{+}$channel inhibitor with $\mathrm{E}_{\max }$ values $(\%)$ of $81.5 \pm 3.5$ vs. control $98.7 \pm 0.5(p<0.001)$ and $(p<0.001)$. Potassium channel blockers cannot inhibit vasodilatation, suggesting that piperitenone oxide vasodilatation may be unrelated to the activation of potassium channels. 


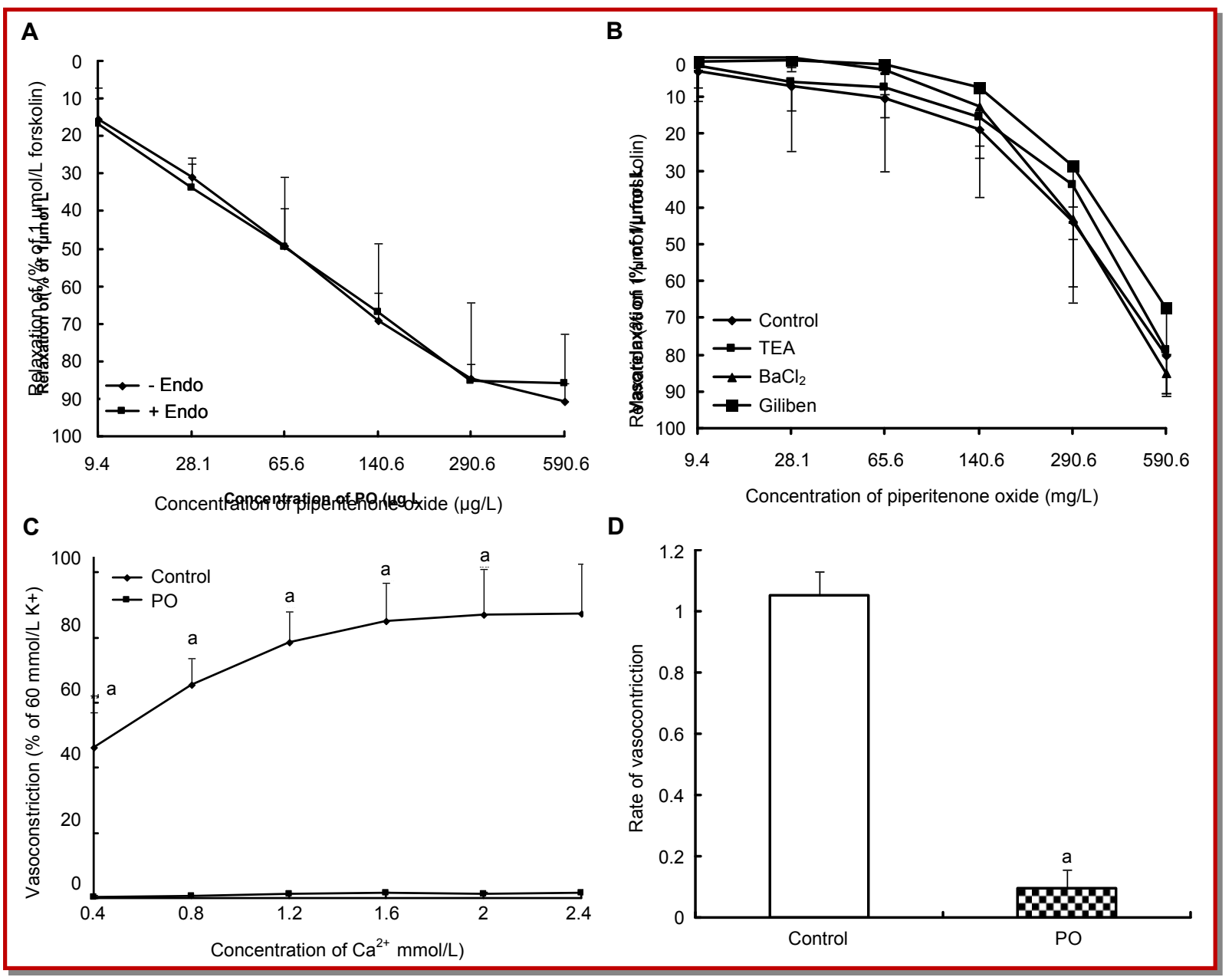

Figure 4: (A) Effect of piperitenone oxide (PO)-induced relaxation in phenylephrine procontracted rat aortic rings with endothelium +endo) and without endothelium (-endo) of SD rats, $\mathrm{n}=3$; (B) Effect of $\mathrm{K}^{+}$channels inhibitors on piperitenone oxideinduced relaxation in rat aortic rings without endothelium (Control), $3 \mathrm{mM}$ TEA (TEA), $10 \mu \mathrm{M}$ gliben (gliben), and $100 \mu \mathrm{M} \mathrm{BaCl} 2$ $\left(\mathrm{BaCl}_{2}\right)$ was pre-incubated respectively, and the inhibitory effects on vasoconstractions were compared to the control groups, $\mathrm{n}=3$; (C) Effect of piperitenone oxide on dose-response curves of $\mathrm{CaCl}_{2}$ in $\mathrm{KCl}(60 \mathrm{mM})$ depolarized rat aortic rings with removed endothelium, $60 \mathrm{mM} \mathrm{KCl}$-induced contraction was used as 100\%, n =5, ap $<0.01$ vs. Control; (D) Effect of piperitenone oxide on intracellular calcium release induced by phenylephrine in rat aortic rings without endothelium, $n=5$, a $p<0.01 v s$. control

\section{Effect on extracellular calcium-induced contraction}

To clarify the effect of piperitenone oxide on concentration-response curves of $\mathrm{CaCI}_{2}$, aortic ring samples denuded of endothelium were exposed to $\mathrm{Ca}^{2+}$-free $\mathrm{K}-\mathrm{H}$ solutions containing $\mathrm{KCl}$, the cumulative addition of $\mathrm{Ca}^{2+}$ induced a concentration-dependent aortic ring contraction. The $\mathrm{CaCl}_{2}$ dose-response curve was significantly down, on concentration-response curves of $\mathrm{CaCl}_{2}$. The difference between the pre-incubation of rings with piperitenone oxide and that without piperitenone oxide was significant $(p<0.01)$, as shown in Figure 4.

\section{Effect on intracellular calcium release}

The addition of phenylephrine $(1 \mu \mathrm{M})$-induced a small tonic contraction elicited mainly by intracellular $\mathrm{Ca}^{2+}$ release from the endoplasmic reticulum $\mathrm{Ca}^{2+}$ stores of smooth muscles, when aorta rings were exposed to $\mathrm{Ca}^{2+}$ -free $\mathrm{K}-\mathrm{H}$ solution, piperitenone oxide $(0.27 \mathrm{~g} / \mathrm{L})$ significantly reduced ring contraction induced by $\mathrm{PE}$ in a $\mathrm{Ca}^{2+}$-free K-H buffer $(\mathrm{p}<0.001)$, as shown in Figure 4.

\section{Discussion}

The present study revealed the significant antihypertensive activity of piperitenone oxide in SHR. Piperitenone oxide lowered the systolic blood pressure and serum ET-1 level in SHR and relaxed the vascular smooth muscle tone in an endothelium-independent manner by regulating the balance between both $\mathrm{Ca}^{2+}$ influx and $\mathrm{Ca}^{2+}$ release in isolated rings of rat aorta. Blood pressure is controlled, in part, by the metabolic homeostasis of endothelium-derived factors, including vasoconstrictor ET-1 and vasodilator NO. Endothelial 
dysfunction correlated with essential hypertension, diabetes, and atherosclerosis is usually characterized by the repression of NO and ET-1 (Ouvina et al., 2001; Perticone et al., 2001). In the current work, given that the norepinephrine-exposed tissue model suggests the endothelium-dependent activity of piperitenone oxide against vasoconstriction, we determined the piperitenone oxide effect on ET-1 secretion and NO production by norepinephrine-exposed BAECs. Cell-based assessment demonstrated that norepinephrine-augmented ET -1 secretion by BAECs was dose-dependently attenuated by piperitenone oxide. Although the precise physiological and pathophysiological roles played by ET-1 remain uncertain, ET-1 is defined as a local autocrine and paracrine factor rather than as a circulating endocrine hormone. It can induce vasoconstriction by binding both ET-A and B receptors on the vascular smooth muscle cells (Maguire and Davenport, 2015). Extracellular calcium entry through voltage-gated $\mathrm{Ca}^{2+}$ channels and intracellular $\mathrm{Ca}^{2+}$ release from the sarcoplasmic reticulum initiate and maintain the contraction of vascular smooth muscle cell. The conjugation of ET-1 to its receptor reportedly elevates extracellular calcium influx and intracellular calcium release via the inositol 1, 4, 5-trisphosphate signaling pathway (Maguire and Davenport, 2015). The level of ET-1 mRNA in endothelial cells can be increased by adrenaline (Peng et al., 2015), which was apparently inhibited by a selective $a_{1}$-adrenoceptor antagonist prazosin but not by the $\alpha_{2}$-adrenoceptor antagonist yohimbine or the $\beta$-adrenoceptor antagonist propranolol (Marasciulo et al., 2006). Thus, the attenuating effect of piperitenone oxide on norepinephrineaugmented ET-1 secretion may involve a $\mathrm{a}_{1}$-adrenoceptor mediated pathway. The endothelial cell molecule that piperitenone oxide binds to requires further research.

In the present study, results showed that piperitenone oxide induced a dose-dependent vasodilator effect in aortic rings pre-contracted with phenylephrine. Piperitenone oxide-induced relaxation was also unaffected by the endothelium, thereby demonstrating that the vasoactive piperitenone oxide acted directly on vascular smooth muscle cells.

We also showed for the first time the vasorelaxant activity of piperitenone oxide in rat aorta. We studied the ability of this compound, accumulatively added to the organ-bath medium, to induce a relaxation of contractions. Data clearly showed that piperitenone oxide can relax, in a concentration-dependent manner, the contractions induced by phenylephrine in rat aortic rings with functional endothelium.

\section{Conclusion}

Piperitenone oxide extract inhibits blood pressure in hypertensive rats. The responsible mechanisms appear to be related to the modulation of ET-1 levels and relaxation, which is associated with interfering intracellular calcium homeostasis by blocking $\mathrm{Ca}^{2+}$ influx, as well as intracellular $\mathrm{Ca}^{2+}$ release.

\section{Ethical Issue}

All procedures were performed in conformity with the Guidelines and Authorization for the Use of Laboratory Animals (Ministry of Health, China Government).

\section{Acknowledgement}

This research was supported by the Natural Science Foundation of China (grant no. 81060350)

\section{References}

Bhatt DL, Kandzari DE, O'Neill WW, D'Agostino R, Flack JM, Katzen BT, Leon MB, Liu ML, Mauri L, Negoita M, Cohen SA, Oparil S, Rocha-Singh K, Townsend RR, Bakris GL. A controlled trial of renal denervation for resistant hypertension. N Engl J Med. 2014; 370: 1393-01.

Editorial board of Flora Xinjiangensis. Flora Xinjiangensis. Urumqi: Xinjiang Science and Technology Publishing House, 2004, p 327.

Guo YT, Lan W, Wu YN, Geng Z. The research of antihypertensive effect on spontaneously hypertensive rats by volatile oil from ziziphora clinopdioides lam. J Xinjing Med Univ. 2014; 37: 257-60.

Havenga MJE, VanDam B, Groot BS, Grimbergen JM, Valerio D, Bout A, Quax PH. Simultaneous detection of NOS-3 protein expression and nitric oxide production using a flow cytometer. Anal Biochem. 2001; 290: 283-91.

Lahlou S, Carneiro-Leao RF, Leal-Cardoso JH, Toscano CF. Cardiovascular effects of the essential oil of mentha $x$ villosa and its main constituent, piperitenone oxide, in normotensive anaesthetised rats: Role of the autonomic nervous system. Planta Med. 2001; 67: 638-43.

Li YL, Wang LM, Jiang Y, Li XY, Zhang M, Hu N. Prevalence of hypertension among Chinese adults in 2010. Chin J Prev Med. 2012; 46: 409-13.

Ling S, Zhang HP, Zhang DD, Zhang LJ, Ka B. Characterizing effects of solvent specific Mori Ramulus components on rat platelet aggregation, vascular tension and macrophage nitrite production. China J Chinese Materia Medica. 2010; 35: $42-46$.

Montagnani M, Chen H, Barr VA, Quon MJ. Insulin-stimulated activation of eNOS is independent of $\mathrm{Ca}^{2+}$ but requires phosphorylation by Akt at Ser1179. J Biol Chem. 2001; 276: 30392-98.

Maguire JJ, Davenport AP. Endothelin receptors and their antagonists [J]. Semin Nephrol. 2015; 35: 125-36.

Marasciulo FL, Montagnani M, Potenza MA. Endothelin-1: The 
yin and yang on vascular function. Curr Med Chem. 2006; 13: 165516-65.

Ouvina SM, La Greca RD, Zanaro NL, Palmer L, Sassetti B. Endothelial dysfunction, nitric oxide and platelet activation in hypertensive and diabetic type II patients. Thromb Res. 2001; 102: 107-14.

Peng G, Li S, Hong W, Hu J, Jiang Y, Hu G, Zou Y, Zhou Y, Xu $\mathrm{J}$, Ran P. Chronic hypoxia increases intracellular $\mathrm{Ca}^{2+}$ concentration via enhanced $\mathrm{Ca}^{2+}$ entry through receptoroperated $\mathrm{Ca}^{2+}$ channels in pulmonary venous smooth muscle cells. Circ J. 2015; 79: 2058-68.

Perticone F, Ceravolo R, Pujia A, Ventura G, Iacopino S, Scozzafava A, Ferraro A, Chello M, Mastroroberto P, Verdecchia P, Schillaci G. Prognostic significance of endothelial dysfunction in hypertensive patients. Circulation 2001; 104: 191-96.

Senejoux F, Demougeot C, Kerram P, Aisa HA, Berthelot A, Bevalot F, Girard-Thernier C. Bioassay-guided isolation of vasorelaxant compounds from Ziziphora clinopodioides Lam. (Lamiaceae). Fitoterapia 2012; 83: 377-82.

Sousa PJ, Magalhães PJ, Lima CC, Oliveira VS, Leal-Cardoso $\mathrm{JH}$. Effects of piperitenone oxide on the intestinal smooth muscle of the guinea pig. Braz J Med Biol Res. 1997; 30: 78791.
Sousa PJC, Linard CFBM, Azevedo-Batista D, Oliveira AC, Coelho-de-Souza AN, Leal-Cardoso JH. Antinociceptive effects of the essential oil of Mentha $x$ villosa leaf and its major constituent piperitenone oxide in mice. Braz J Med Biol Res. 2009; 42: 655-59.

Verdian RM. Essential oil composition and biological activity of Ziziphora clinopodioides Lam. From Iran. Am-Eurasian J Sustain Agric. 2008; 2: 69-71.

World Health Organization. Global health risks: Mortality and burden of disease attributable to selected major risks, 2009.

Xue YL, Shi HX, Ferid M, Ka B. Vasodilatory effects of cinnamaldehyde and its mechanism of action in the rat aorta. Vasc Health Risk Manag. 2011; 7: 273-80.

Zhang HP, Li YJ, Zhang DD, Ka B. Vasodilatatory effect and mechanism of $\mathrm{CH}_{2} \mathrm{Cl}_{2}$ extract of flos magnoliae on isolated rat thoracic aorta. Chin J Pathophysiol. 2010; 26: 1689-94.

Zhang DD, Ling S, Zhang HP, Ka B. The inhibitory effect of different fractions from Nigellae Semen in vitro on nitrite accumulation, platelet aggregation and vasoconstriction. Chin Tradit Patent Med. 2013a; 35: 1376-80.

Zhang HP, Tian G, Yang T, Chen L. Vasodilatation mechanism of Magnolin in isolated rat thoracic aorta and its toxicity in rat renal cells NRK. Chin J Exp Tradit Med Formulae. 2013b; 19: 209-12.

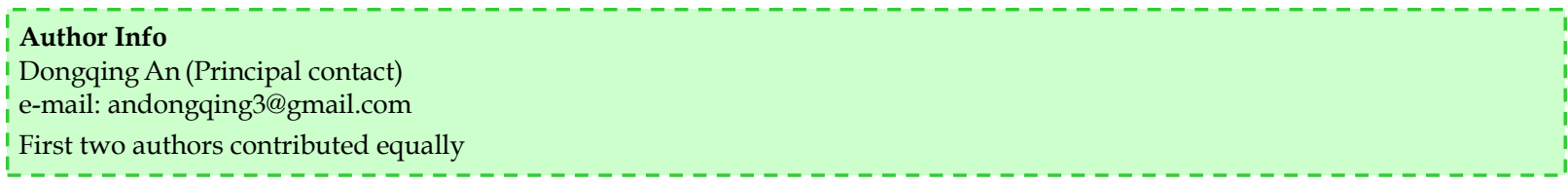

